\title{
Aportaciones para un estado de la cuestión sobre pertinencia en la formación profesional turística
}

Fredy-Ismael González-Fonseca, Ana-Leticia Tamayo-Salcedo y Elva-Esther Vargas-Martínez

\section{RESUMEN}

Existe un número considerable de trabajos sobre pertinencia en la educación superior. Sin embargo, es reducido el número de estudios sobre pertinencia en la formación de profesionales en turismo de México. El objetivo del trabajo es presentar aportaciones para el estado de la cuestión sobre pertinencia en la formación profesional turística, en el período 2000-2015, a través del análisis de artículos indexados publicados. El primer momento es el procedimiento metodológico; el segundo es un recorrido sobre las interpretaciones del término pertinencia en la educación superior; el tercero es el resultado de una investigación documental sobre la postura de los académicos acerca de la pertinencia en la formación de profesionales en turismo y, la última sección, presenta las conclusiones y futuras líneas de investigación. Entre los resultados más relevantes, se destaca la constante preocupación por la relación universidadsociedad y las tensiones existentes entre la formación de profesionales en turismo y el mundo del trabajo.

Palabras clave: pertinencia social, Formación profesional, Turismo, México.

Fredy-Ismael González-Fonseca fredy_gf@yahoo.com.mx Colombiano. Doctor en Estudios Turísticos, Centro de Investigaciones en Estudios Turísticos (CIETUR), Facultad de Turismo y Gastronomía de la Universidad Autónoma del Estado de México (UAEM), Maestro en Administración, Universidad Nacional Autónoma de México (UNAM). Profesor Investigador de Carrera de la Universidad de Quintana Roo, Unidad Académica Chetumal, México. Temas de investigación: formación profesional en turismo, ecoturismo y turismo sustentable.

\section{Ana-Leticia Tamayo-Salcedo}

altamayos@uaemex.mx Mexicana. Doctora en Ciencias de la Educación, Instituto Superior de Ciencias de la Educación del Estado de México. Profesora investigadora de tiempo completo, Centro de Investigación y Estudios Turísticos (CIETUR), Facultad de Turismo y Gastronomía, Universidad Autónoma del Estado de México (UAEM). Temas de investigación: construcción del conocimiento y formación profesional en turismo. sustentabilidad, responsabilidad social, empresas turísticas. 


\section{Contribuições para um estado da questão sobre pertinência na formação profissional turística}

\section{RESUMO}

Existe um número considerável de trabalhos sobre pertinência na educação superior. Porém, é reduzido o número de estudos sobre pertinência na formação de profissionais em turismo do México. O objetivo do trabalho é apresentar contribuições para o estado da questão sobre pertinência na formação profissional turística, no período 2000-2015, através da análise de artigos indexados publicados. O primeiro momento é o procedimento metodológico; o segundo é uma análise das interpretações do termo pertinência na educação superior; o terceiro é o resultado de uma pesquisa documental sobre a postura dos acadêmicos em relação a pertinência na formação de profissionais em turismo, e a última seção apresenta as conclusões e futuras linhas de pesquisa. Entre os resultados mais relevantes, se destaca a constante preocupação pela relação universidade-sociedade e as tensões existentes entre a formação de profissionais em turismo e o mundo do trabalho.

Palavras chave: pertinência social, formação profissional, turismo, México.

\section{Contributions for a state of affairs about pertinence in tourism professional training}

\section{ABSTRACT}

There is a considerable body of work about pertinence in higher education. However, the amount of studies about pertinence in the field of training for tourism professionals in Mexico is small. The purpose of this article is to present contributions to the state of affairs about pertinence in tourism professional training during the period 2000-2015, through the analysis of published indexed articles. The first step is a description of the methodological procedure; the second is a tour on the interpretations of the term relevance in higher education; the third is the result of a documentary research on the position of academics about pertinence in the training of professionals in tourism, and the last section presents the conclusions and future lines of research. Among the most relevant results we can point out the constant concern for the university-society relationship and the existing tensions between the training of professionals in tourism and the world of work.

Key words: social pertinence, professional training, tourism, Mexico.

Recepción: 29/12/16. Aprobación: 8/12/17. 


\section{Introducción}

La educación alberga un complejo entramado de caminos que conducen a un desafío, el conocimiento. En este sendero con obstáculos, aparecen varios actores que desde diferentes escenarios dan forma a la educación superior en todo el mundo; organismos nacionales e internacionales encargados de formular políticas que coadyuvan a mejorar su calidad trabajando en la complicada pero satisfactoria misión de formar para el desempeño profesional.

La universidad, como centro formador, diseña y promueve programas educativos a partir de las necesidades del entorno que les rodea, o al menos en teoría ese es el propósito; esas necesidades se presentan en varias direcciones: sociales, económicas, políticas, culturales y ambientales.

En 1998, con ocasión de la Conferencia Mundial de la Educación celebrada en París, uno de los puntos clave en las mesas de trabajo fue el tema de la pertinencia en la educación superior; diez años después en 2008, un grupo de investigadores liderados por Tünnermann (2008) retomó el tema a partir del análisis del estado de la educación en América Latina y el Caribe, rescatando los compromisos llevados a cabo en 1998, su cumplimiento y las responsabilidades de los diferentes actores.

El balance fue negativo, y el debate tomó otra dirección, centrándose en la manera como debía repensarse drásticamente la educación en su concepción, objetivos y alcance; los niveles educativos debían elevarse, las instituciones fortalecerse; debían crearse nuevos instrumentos educativos a lo largo y ancho de la sociedad, y la investigación de carácter limitativo, expandirse (Cooper, 2012; Vessuri, 2008; Pernas et al., 2009). La pertinencia en la educación superior, estuvo ausente en la composición temática de conferencias, simposios, conversaciones y ponencias a cargo de investigadores mexicanos y extranjeros en el marco del XIII Congreso Nacional de Investigación Educativa, uno de los más importantes espacios de discusión latinoamericanos en la materia (Ordorika, 2015).
De ahí que el debate de la pertinencia y su rol en la formación de profesionales para el turismo no se ha iniciado aún, ya que la velocidad de las dinámicas propias del sector turismo, no ha dado lugar a que se abran los espacios de investigación y reflexión sobre el tema desde las instituciones de educación superior (IES). Cabe señalar que en la década de los setenta, el sistema de educación superior en México reconocía dentro de sus tres retos fundamentales para su desarrollo, ofrecer programas educativos con calidad y pertinencia accesibles para todos los grupos sociales (Mungaray et al., 2016).

El presente trabajo tiene como objetivo dar a conocer las aportaciones para un estado de la cuestión sobre pertinencia en la formación profesional turística, en el periodo 2000-2015. Se toman en consideración las diversas interpretaciones del término pertinencia en la educación superior y a la postre una investigación documental sobre la postura de la comunidad científica acerca de la pertinencia en la formación de profesionales en turismo.

Para su construcción se consideraron las contribuciones de investigaciones publicadas en revistas indexadas relacionados con el objeto del trabajo. Se identificaron posturas, problemas planteados, hipótesis que se privilegian, propósito de las investigaciones, métodos utilizados y principales resultados.

Cabe señalar que la formación profesional en turismo en Europa tiene una larga historia e influencia en la región latinoamericana; e inclusive la tradición en investigación, como lo confirman estudios en revistas indexadas, relativos a la pertinencia de los programas que forman profesionales en turismo.

El documento está dividido en cuatro secciones; la primera parte es el procedimiento metodológico que guía la construcción del mismo; la segunda parte un recorrido sobre las interpretaciones del término pertinencia en la educación superior en Latinoamérica, región con significativas publicaciones arbitradas; la tercera aborda los resultados de investigaciones académicas realizadas por investigadores del turismo 
en Europa, región de referencia, tradición y publicaciones acerca de la pertinencia en la formación de profesionales en turismo; la última parte, a manera de reflexiones, presenta las conclusiones y lo que pueden constituir futuras líneas de investigación a partir de lo indagado.

\section{Procedimiento metodológico}

Las fuentes de investigación del presente documento están constituidas por un conjunto de revistas especializadas que, entre sus temas, han considerado entidades como pertinencia, formación profesional, educación superior y formación profesional en turismo. La selección de los repositorios se hizo en función de las disciplinas donde se enmarca la investigación; posteriormente se dispusieron las revistas científicas para analizar. El criterio considerado fue precisar dos tipos de revistas, a partir del área de conocimiento y la disciplina (Picazo y Moreno, 2012). De esta manera, desde las ciencias sociales y administrativas se consultaron revistas de: administración, estudios organizacionales, investigación educativa, sociología y turismo. Desde la educación y las humanidades se consideraron revistas de: ciencias de la educación, docencia superior, educación superior y pedagogía. El cuadro 1 da cuenta del número de artículos seleccionados y el repositorio al que pertenecen las revistas.

Inicialmente, el periodo de análisis comprendía diez años (2005-2015), pero en razón al bajo número de publicaciones fue necesario ampliar el margen, considerando el periodo (2000-2015). El cuadro 2 muestra el número de revistas indexadas contempladas en el estudio. En la construcción del documento, la selección de artículos en revistas indexadas privilegió las regiones de Latinoamérica y Europa. La elección de las regiones obedece, en el caso de la primera, a que México como país latinoamericano es un referente histórico en términos de formación en turismo al iniciar el primer programa educativo a nivel técnico en 1953, con la Escuela Mexicana de Turismo, y a nivel licenciatura en 1959, con la Universidad Autónoma del Estado de México (UAEM). La segunda región, Europa, obedece a que la mayor producción científica sobre pertinencia en la formación profesional en turismo, se ubica en autores de dicha procedencia (Tribe, 2005a) y de ahí la existencia de revistas especializadas en temas relacionados con el currículo, modelos de planificación, perfiles de egreso y la oferta educativa en turismo.

Siguiendo el procedimiento descrito, se analizó por separado cada una de las revistas seleccionadas, verificando qué artículos contenían la temática referida, evaluando posturas, problemas planteados, hipótesis que privilegian, propósito de las investigaciones, métodos utilizados y principales resultados. De este modo se obtuvo una base de 70 artículos publicados en 36 revistas diferentes. Una vez construida la base de datos, se analizó cada artículo a partir de una ficha en la que se incluía la información anterior. El cuadro 3 presenta de manera sintética las categorías sobre las cuales se hizo énfasis, los artículos relacionados y el porcentaje que representa cada categoría. En las revistas incluidas para el estudio, pertinencia fue una de las categorías de análisis que mayores resultados arrojó.

Cuadro 1. Relación de repositorios y artículos analizados

\begin{tabular}{|c|c|c|c|c|c|c|}
\hline Scopus & Scielo & Taylor\&Francis & Ebsco & Redalyc & $\begin{array}{c}\text { Sage } \\
\text { Journals }\end{array}$ & $\begin{array}{c}\text { Research } \\
\text { Gate }\end{array}$ \\
\hline 20 & 11 & 10 & 10 & 8 & 6 & 5 \\
\hline
\end{tabular}

Fuente: elaboración propia. 


\section{Cuadro 2. Listado de revistas incluidas en el estudio}

\begin{tabular}{|c|c|c|}
\hline & Revista & Núm. de \\
\hline 1. & Administración y Organizaciones & 1 \\
\hline 2. & Annals of Tourism Research & 2 \\
\hline 3. & Association for Tourism in Higher Education & 5 \\
\hline 4. & CIENCIA Ergo-Sum & 1 \\
\hline 5. & Ecos de Economía & 1 \\
\hline 6. & Educación Médica Superior & 1 \\
\hline 7. & Educación Superior y Sociedad & 2 \\
\hline 8. & Educación y Educadores & 1 \\
\hline 9. & Educatio siglo XXI & 2 \\
\hline 10. & Estudios Sociales & 1 \\
\hline 11. & Estudios Sociológicos & 1 \\
\hline 12. & Gestión turística & 2 \\
\hline 13. & International Journal of Hospitality Management & 1 \\
\hline 14. & International Review of Education & 1 \\
\hline 15. & Journal of Geography in Higher Education & 1 \\
\hline 16. & $\begin{array}{l}\text { Journal of Hospitality, Leisure, Sport \& Tourism } \\
\text { Education }\end{array}$ & 4 \\
\hline 17. & Journal of Teaching in Travel \& Tourism & 5 \\
\hline 18. & Journal of Travel Research & 5 \\
\hline 19. & Journal of Vocational, Education and Training & 1 \\
\hline 20. & Management and Education & 1 \\
\hline 21. & Multiciencias & 1 \\
\hline 22. & Perfiles Educativos & 2 \\
\hline 23. & Revista Actualidades Investigativas en Educación & 1 \\
\hline
\end{tabular}




\begin{tabular}{|ll|c|}
\hline 24. & Revista de Estudios Sociales & 1 \\
\hline 25. & Revista de la Educación Superior & 3 \\
\hline 26. & $\begin{array}{l}\text { Revista del Instituto de Estudios en Educación } \\
\text { Universidad del Norte }\end{array}$ & 1 \\
\hline 27. & Revista Educación Química & 1 \\
\hline 28. & Revista Espacio Abierto & 2 \\
\hline 29. & Revista Española de Investigaciones Sociológicas & 1 \\
\hline 30. & Revista Española de Pedagogía & 3 \\
\hline 31. & Revista Iberoamericana de Educación & 7 \\
\hline 32. & Revista Iberoamericana de Educación Superior & 1 \\
\hline 33. & Revista Nómadas & 70 artículos \\
\hline 34. & Revista Pedagogía Universitaria & 1 \\
\hline 35. & Revista Unipluriversidad & Tourism Management \\
\hline 36. & Total & 1 \\
\hline
\end{tabular}

Fuente: elaboración propia.

\section{Cuadro 3. Artículos por categoría de análisis}

\begin{tabular}{|l|c|c|}
\multicolumn{1}{|c|}{ Categoría } & Número de artículos & Porcentaje \% \\
\hline Pertinencia & 32 & $46 \%$ \\
\hline Formación profesional & 8 & $11 \%$ \\
\hline Educación superior & 10 & $14 \%$ \\
\hline Formación profesional en turismo & 20 & $29 \%$ \\
\hline Total & 70 & $100 \%$ \\
\hline
\end{tabular}

Fuente: elaboración propia. 
A continuación, se presentan los resultados del análisis documental en dos secciones; la primera abarca las interpretaciones conceptuales del término pertinencia en el marco de la educación superior iniciando con el origen etimológico. La segunda sección aborda la producción científica en torno a la pertinencia de la formación profesional en turismo, objeto de estudio del presente estado de la cuestión.

\section{Marco conceptual de pertinencia en educación superior}

Etimológicamente el término pertinencia se deriva de dos partes: del prefijo "per" que se traduce como "por completo" y el verbo tenere que es sinónimo de "sostener". Pertinencia es la cualidad de pertinente; se trata de un adjetivo que hace mención a lo perteneciente o correspondiente a algo o aquello que viene a propósito (Pérez y Merino, 2010).

En el ámbito educativo, en 1998, con ocasión de la Conferencia Mundial de la Educación celebrada en París, uno de los puntos clave en las mesas de trabajo fue el tema de la pertinencia en la educación superior. Según la United Nations Educational, Scientific and Cultural Organization (UNESCO) el concepto de pertinencia en educación superior atiende a lo siguiente: "La pertinencia en educación superior se considera primordialmente en función de su cometido y su puesto en la sociedad, de sus funciones con respecto a la enseñanza, la investigación de los servicios conexos, y de sus nexos con el mundo del trabajo en sentido amplio, con el estado y la financiación pública y sus interacciones con otros niveles y formas de la educación" (UNESCO, 1998: 19).

Al respecto Malagón (2009b), a partir de un análisis conceptual sobre pertinencia de la educación superior, reconoce la postura de diversos autores y formula una serie de enfoques para su estudio. El primer enfoque economicista (Malagón, 2006) está basado en los planteamientos de Gibbons (1998) y su propuesta en la necesidad de que las universidades se vinculen estrechamente con el sector productivo.
El segundo enfoque de corte social surge a partir de los trabajos de Vessuri (1996), García (1997), Tünnermann (2000) y De Ketele (2008), que enfatizan la naturaleza social de la vinculación universidad y su entorno. El último es el enfoque integral que resulta como síntesis de los anteriores, introduciendo al currículum como un eje central de la pertinencia (Corzo y Marcano, 2009; López, 2007). Aquí se retoma el concepto expresado por Kemmis (1993) sobre la doble función del currículo. En el enfoque integral, Malagón (2009b) hace referencia a la pertinencia institucional, social, económica, pedagógica, externa e interna y señala que todas tienen un significado.

El enfoque economicista de pertinencia, implica la contribución de productos hacia la economía nacional en pro del desarrollo económico; desde un enfoque pragmático, Gibbons (1998: 2) recalca que "los imperativos económicos barrerán con todo lo que se les oponga, y si las universidades no se adaptan, se las dejará de lado".

La Organización para la Cooperación y el Desarrollo Económico (OCDE, 1997) enfatiza que, para promover la pertinencia de la formación profesional, es necesaria la inclusión de representantes de los sectores económicos y sociales en las instancias de dirección de las IES. Una tarea primordial es la definición de los planes de estudio conjuntamente entre instituciones educativas, negocios e industrias, además de otras acciones de vinculación con el contexto socioeconómico (Camarena y Velarde, 2010; Lombana, 2012; Mora y De Oliveira, 2012). A partir de este enfoque predominan los estudios sobre formación profesional en turismo, realizados por el Banco Interamericano de Desarrollo (BID, 2009), la Universidad La Salle (2012), la Organización Mundial del Turismo (OMT, 2009) y el Ministerio de Educación de Argentina (2013).

En cuanto al enfoque social de pertinencia en educación superior, la UNESCO (1998) establece que es la adecuación entre lo que la sociedad espera de 
ellas, y lo que las instituciones realmente hacen; esto significa que deben involucrar aspectos como: normas éticas, imparcialidad política, capacidad crítica y una articulación con las demandas de una sociedad y el mundo del trabajo.

Por su parte Tünnermann (2000), sobre la base conceptual del término pertinencia de la educación superior, destaca una serie de aspectos que denotan la capacidad de las instituciones para conectarse con una diversidad de asociados en varios niveles y trabajar con ellos de manera creativa. El autor sostiene que las universidades deberán establecer múltiples y diferentes tipos de vinculaciones con la sociedad que las rodea. Por lo cual la pertinencia debe ser analizada a partir de aspectos como los procesos de selección de la información, desde la promoción de un nuevo proyecto educativo centrado en el aprendizaje; la producción y organización del conocimiento; la nueva concepción de las profesiones, desde la función social de la universidad; la dimensión nacional, regional e internacional de la educación, y la evaluación institucional (Tünnermann, 2006; Beltrán et al., 2014; Garrocho, 2012; Gottifredi, 1996; Navarro, 1997; Malagón, 2003; Schleicher, 2008). La tesis del autor manifiesta que la pertinencia de la educación superior debe examinarse bajo el amplio concepto de pertinencia social, sin reducir su análisis a la respuesta que ésta debe dar a las demandas de la economía o del sector laboral (Tünnermann y De Souza, 2003).

Pérez (2007) considera que la pertinencia social relaciona a la educación superior con la sociedad planteando la necesidad de una evaluación externa en términos de expectativas y logros públicos. El concepto de pertinencia obliga a la universidad a responder ante la sociedad sobre lo que la institución universitaria genera como producto (Naidorf et al., 2007; Tünnermann, 2007); "la pertinencia se vincula con el principio de responsabilidad, y permite de este modo romper la posibilidad de un cerco de autoaislamiento universitario incompatible con la misma tradición latinoamericanista de una universidad pública en armonía con las necesidades de la sociedad civil y con las condiciones de su progreso" (Pérez, 2007: 5).

Vessuri (2008) explica que pertinencia es la coincidencia entre lo que las IES hacen y lo que la sociedad espera de ellas en estricto sentido. Hace énfasis en el rol de la educación superior en la sociedad, el cual comprende participación, acceso, enseñanza y aprendizaje para los estudiantes; desde luego debe cumplir con la función de centro de investigación, de responsabilidad social con impacto no sólo en el sector laboral, sino también en la solución de problemas humanos urgentes alrededor de la población, el medio ambiente, la paz y la defensa de los derechos humanos (Días-Sobrinho et al., 2008; Orozco, 2010; Chavoya y Reynaga, 2011; Núñez, 2010; Nieto, 2014; Salgado, 2004).

Gómez (1998) relaciona el concepto de pertinencia social a partir de ocho aspectos de la siguiente manera:

- Pertinencia institucional que involucra la evaluación de los proyectos, objetivos y necesidades de las IES.

- Pertinencia política que significa trabajar para construir soluciones.

- Pertinencia pedagógica, es decir innovar desde lo educativo para responder a las necesidades de la juventud y la sociedad.

- Pertinencia en la formación integral del estudiante, que debe considerar los valores, la ética y el sentido de pertenencia a una comunidad.

- Pertinencia de la equidad social del desarrollo.

- Pertinencia en términos de ampliación social de cobertura y cultural que involucra tres aspectos: a) fortalecimiento de la identidad nacional, rescate y valoración del patrimonio cultural, b) promoción de múltiples formas de expresión, y c) oferta cultural libre e incluyente.

- Pertinencia con el resto del sistema educativo. 
- Pertinencia con el sector productivo, relacionada con las demandas de la economía.

Lo anterior denota un desbordamiento de aspectos en el enfoque social de la pertinencia, donde no sólo implica una dimensión social y económica, sino también cultural e institucional; el currículum entra en escena mediatizando la articulación de la relación universidad-sociedad.

El enfoque integral de pertinencia conjuga los aspectos anteriores en cuanto a ser capaz la universidad de explicar la relación teoría-práctica y la relación universidad-sociedad, es decir, examinar la naturaleza social, cultural, económica, pedagógica, epistemológica, conceptual, teleológica e investigativa, entre otras. Donde la universidad no solamente se adapte o adecue, sino que pueda ser una institución crítica y capaz de incidir en el entorno.

Derivado del anterior recorrido conceptual sobre pertinencia, se puede advertir que desde un enfoque economicista la pertinencia se juzga a partir de las contribuciones que privilegien el desarrollo económico nacional; en otras palabras, las IES al servicio del aparato productivo. Por otro lado, desde un enfoque social, el concepto de pertinencia responde directamente a los esquemas de desarrollo de la sociedad en que se inserta cada organización educativa, donde las IES son protagonistas de los procesos sociales, económicos y políticos con capacidad de diálogo con el medio y consigo misma. Y desde un enfoque integral, la pertinencia debe ser capaz de participar, acompañar y liderar los cambios del entorno.

Se puede mencionar que la pertinencia no es un concepto estático, se re-elabora o re-construye constantemente, dados los cambios permanentes de la misma sociedad y sus dinámicas; y se va adaptando a un ambiente social específico pero en continua evolución.

A partir del análisis de las investigaciones de los autores que publicaron en revistas indizadas, y las publicaciones de los programas multilaterales sobre pertinencia, para efectos de la presente investigación el concepto que se asume emerge desde un enfoque social. La construcción da como resultado lo siguiente: pertinencia es la capacidad de respuesta interna y externa de las IES de carácter público y privado a las necesidades (de naturaleza cambiante) de una sociedad en continua transformación, en su concepción participan agentes internos y externos, y está acotada al momento socio histórico de la nación en la cual están insertas las instituciones educativas.

La pertinencia es de naturaleza compleja, donde se liga lo interno y externo de la universidad. Por ello es importante analizar la producción científica en torno a la pertinencia en la formación profesional turística.

\section{Producción científica en torno a la pertinencia en la formación profesional en turismo}

Los estudios sobre turismo son relativamente recientes. Mientras que otras disciplinas como la química, la medicina, la matemática o la geografía han sido objeto de análisis desde la más remota antigüedad, los viajes "de placer" comienzan a estudiarse de forma científica hasta el periodo de entreguerras (1919-1939).

La definición académica que sobre turismo se ha aceptado universalmente, se debe a dos profesores economistas suizos: Walter Hunziker y Kart Krapf (1942), en plena II Guerra Mundial, los autores establecen que: "Turismo es el conjunto de relaciones y fenómenos que se producen como consecuencia del desplazamiento y estancia temporal de personas fuera de su lugar de residencia, siempre que no esté motivado por razones lucrativas" (Hunziker y Krapf, 1942: 13). Desde una mirada sociaológica, para Urry y Larsen (2011), el turismo es una actividad de ocio que presupone su opuesto, es decir, el trabajo regulado y organizado. Es una manifestación de cómo el trabajo y el ocio se organizan como 
esferas separadas y reguladas por la práctica social en las sociedades modernas (Urry y Larsen, 2011: 4).

La importancia económica y social que empezó a tener el turismo, condujo a buscar la profesionalización de recursos humanos, por ello surge la enseñanza del turismo de manera institucionalizada a través de diversas IES.

De acuerdo con Jafari (2005), durante la primera parte del siglo XX, varias universidades europeas ya habían establecido cátedras de turismo, mientras que en Estados Unidos y otros lugares del mundo lo descubrieron mucho más tarde. Los programas de gestión hotelera fueron pioneros en el currículo y rápidamente se desarrollaron otros programas educativos con orientación a la gestión de negocios, ocio, esparcimiento, e incluso ciencias sociales (Jafari, 2005: 46).

En la región latinoamericana, la formación en turismo tiene una historia de 57 años; en todos los países del continente americano inicia como una disciplina técnica de nivel terciario que con el paso del tiempo vive una transición con programas en el nivel superior.

En México la formación profesional en turismo se inició en 1959 con el surgimiento de la licenciatura en turismo de la UAEM; a partir de los años setenta empieza el boom por establecer carreras en esta área, especialmente en las universidades públicas y autónomas de Nayarit, Guerrero, Baja California y Guadalajara. Una década más tarde tuvo lugar una fuerte expansión de licenciaturas en turismo a lo largo y ancho del territorio nacional, (Gómez, 2012: 99). Cabe señalar que en la década de los años setenta, el sistema de educación superior en México reconocía dentro de sus tres retos fundamentales para su desarrollo ofrecer programas educativos con calidad y pertinencia accesibles para todos los grupos sociales (Mungaray et al., 2016).

Para el análisis de la producción científica en torno a la pertinencia en la formación profesional turística, se considera la región de Europa pues los autores de esta región han privilegiado este tema en sus investigaciones. Es preciso acotar que la formación profesional en Europa está viviendo un proceso de reflexión y cambio para adaptarse a nuevos retos de una economía postindustrial y globalizada, que se adentra en la llamada sociedad del conocimiento (Arellano y Castro, 2013; Escobar, 2003).

En contraste, los procesos de formación profesional en turismo se han visto enmarcados en una disciplina que carece de antecedentes en temas formales (por ejemplo, planificación y desarrollo, administración pública, turismo internacional, entre otros) y de raíces que permitan tener éxito en la gestión de la escuela (Perrenoud, 2005). Otras disciplinas, como la geografía, la economía o la sociología tienen esa claridad demostrada en los resultados de investigaciones de corte científico. Dicha carencia dejó al turismo sin un cuerpo articulado de conocimientos (Goodenough y Page, 1993; Ayikoru et al., 2009; Zagonari, 2009). Los conceptos y las definiciones para explicar el fenómeno turístico surgen en varias direcciones, que utilizan métodos y en ocasiones teorías de otras disciplinas. Sin embargo, es ampliamente aceptado un núcleo de productos o componentes básicos (como transporte, atracciones, alojamiento, alimentos y bebidas) que se traducen en una pérdida de asociación que se identifica como la industria del turismo (Mayaca y Akama, 2007).

Esta pérdida de asociación derivó, entre otras, en la dificultad de evaluar el alcance por el cual la industria del turismo existe como un sector articulado con sus propios requerimientos para la formación de recursos humanos. Esto genera un dilema para las IES, las cuales deben decidir si centrar el currículo sobre la base de los requerimientos de la industria o centrado en el estudiante (Fidgeon, 2010; Alcántara y Zorrilla, 2010; Malagón, 2009a). En palabras de Tribe (2005), un plan de estudios con fines de formación profesional centrado en la industria o un plan que abarque un enfoque más liberal.

La experiencia de potencias como Reino Unido para suplir la demanda educativa en la disciplina 
turística, comprende un proceso de negociación de actores como: empleadores, asociaciones no gubernamentales (por ejemplo the Association of British Travel Agents), reconocidos cuerpos de investigación en la disciplina y el Departamento de Ciencia y Educación (Department of Education and Science). Aun así, el debate se mantiene en torno a decidir el alcance que los programas de formación profesional en turismo requieren para cumplir con las necesidades de la industria.

Airey y Middleton (1995) argumentan que la posesión de una carrera profesional en turismo por sí misma no provee un pasaporte para el ingreso a la profesión; ciertas características de la personalidad, aptitudes gerenciales, habilidades y conciencia, también son muy importantes para la mayoría de los empleadores. Por ello la dificultad para llegar a un amplio acuerdo que permita proporcionar a las IES, el alcance y flexibilidad para emplear diversos elementos en el diseño de planes de estudio de manera particular. Airey y Middleton (1995) afirman que en los noventa las personas que trabajaban en la industria del turismo, no contaban con un grado o título; en algunos casos esa tendencia se mantiene en la actualidad (Walmsley, 2012).

De acuerdo con Gómez (2009), el número de oportunidades a nivel de educación superior, en programas de medio tiempo y tiempo completo disponible para aquellos que buscaban un título relevante en la industria del turismo, proporcionaban una amplia gama de alternativas.

A partir de esa gama de alternativas que comenzó en 1986 y en mayor proporción en los noventa, son varias las aproximaciones que se presentan alrededor del diseño de programas en turismo. Así como nuevos temas aparecen (por ejemplo, el desarrollo sustentable), nuevos espacios deben ser "inventados" en los planes de estudio; además de establecer alianzas internacionales para unir esfuerzos por diseñar y promover un plan de estudios atractivo para aquellos estudiantes de intercambio, con la necesidad de vinculación con el sector productivo (Holloway, 1995; Eber, 2003; Marum y Rosario, 2001; Horng y Lee, 2005).

Botterill (1996), al respecto de la vinculación, reconoce tres tipos de relación entre la industria y la universidad. La primera relación se distingue por un enfoque de plena independencia y competencia entre ambas partes, tomando posturas poco arraigadas y criticas una de la otra. La segunda se traduce en un enfoque cooperativo en el que se encuentran proyectos específicos para beneficio mutuo, donde hay cambios mínimos a las prácticas existentes entre ambas partes. El tercero es el más reciente y se puede describir como un enfoque de alianza estratégica, el cual obliga a las partes a trabajar de manera conjunta para buscar innovar y resolver problemas afines (Solnet et al., 2007). En muchos aspectos, este último enfoque es el más difícil de lograr, pero puede aportar las mayores recompensas para ambas partes. Dadas las presiones competitivas en la educación superior y la necesidad de calidad (Buendía, 2014), la alianza estratégica puede llegar a ser la futura forma prospectiva de la relación industria/educación superior en turismo que se encuentra representada por empresarios y académicos, lo cual no escapa a la percepción de los egresados en torno a la pertinencia (Ernawati y Pearce, 2003; Almonacid et al., 2009; Martínez, 2003; Méndez, 2005).

Botterill y Tribe (2000) argumentan que la posesión de un título de pregrado en turismo ha provocado un debate considerable en la educación superior británica, en donde la producción de investigación reciente sobre el tema se traduce en una amplia comparación de ideas sobre el espacio curricular (Collado et al., 2013). Argumentan que, para el turismo, la ausencia de un paradigma aceptado y la diversidad de posibles empleos, significa que hay un espacio latente para una variedad de configuraciones e inserciones en los planes de estudio.

De acuerdo con Avcikurt (2000), en un estudio realizado en Turquía sobre las previsiones en educación 
y formación en turismo, en términos generales los problemas de la formación superior turística se pueden agrupar en cuatro categorías principales:

1. La política de educación y planificación del turismo

2. Integración y coordinación en la educación turística

3. Colaboración del sector turístico y las IES en turismo

4. Reconocimiento del personal de turismo como profesionales.

Advierte que a medida que crece la industria del turismo, se ha generado una creciente necesidad de una política planificada sobre la base estimada y real del personal capacitado; en esta planificación, la intervención del ministerio de cultura y turismo en asociación con el ministerio de educación es clave en todo el proceso, (Avcikurt et al., 2009; Busby y Huang, 2012).

Tribe (2001), en un estudio sobre los paradigmas en la investigación y el currículo en turismo, clasifica y evalúa las metodologías usadas para el diseño del mismo. Hace un recorrido por los trabajos de Koh (1995) y el estudio The National Liaison Group for Higher Education in Tourism (NLG) (Holloway, 1995), en el cual se destaca el paradigma positivista en la adopción de los planes de estudio. Este método da como resultado un plan de estudios vocacional, basado en la técnica y los medios en lugar de considerar los fines. La producción de graduados con buenas técnicas de negocio es por supuesto un objetivo clave de la formación profesional turística enmarcada en el paradigma positivista (Gunn, 1998).

Tribe (2001) señala que los planes de estudios necesitan desarrollar una sociedad del turismo no sólo para los negocios, sino también para todos los grupos de interés. En otras palabras, los intereses prácticos y emancipadores deben ser considerados, así como los intereses técnicos. Lo cual es coherente con los fines de la pertinencia social de la educación superior, al considerar a la comunidad universitaria, el sector productivo y el estado en su conjunto, (Osorio et al., 2010; Coronado y Estévez, 2016).

Ring et al. (2009) indican que la formación en el ámbito del turismo comenzó a partir de cursos de capacitación para el personal en sectores específicos de la industria; posteriormente estos cursos condujeron al establecimiento de escuelas técnicas y vocacionales, las cuales a su vez han evolucionado en programas de pregrado y posgrado (Busby y Fiedel 2001; Tribe, 2002; Busby, 2003; Airey, 2005).

Ring et al. (2009) examinan las expectativas de los profesionales en turismo y los académicos, en lo concerniente a los contenidos de los planes de estudio de licenciatura en turismo, con el objetivo de identificar y discutir aquellos de gran utilidad para el diseño de un programa. Universidades de Australia, China, Irlanda, Inglaterra, Estados Unidos y Nueva Zelanda fueron consideradas en la muestra para el análisis de contenidos. De acuerdo con la investigación, el énfasis de los contenidos curriculares se da en cuatro categorías, a saber: 1. Administración; 2. Medio ambiente, 3. Naturaleza y ecoturismo; 4. Mercadotecnia turística y turismo social. De los resultados se destaca que el $75 \%$ de los programas tiene un claro enfoque a la administración; el 9\% está centrado en cuestiones medio ambientales, turismo de naturaleza, ecoturismo y áreas afines; el 8\% de los programas está enfocado a la mercadotecnia turística; la categoría de turismo social obtuvo un 8\% de asignación y abarca programas concentrados en temas como diversidad cultural, conservación del patrimonio y una interpretación de las circunstancias locales y regionales (Tan y Morgan, 2001; Craig-Smith y Ding, 2007). Los resultados destacan una clara tendencia de programas centrados en las necesidades del sector productivo.

Airey et al. (2014) hacen énfasis en la necesidad de planear la educación superior en turismo a partir de una organización internacional que convoque 
asociaciones temáticas y éstas a su vez coordinen $\mathrm{y}$ fomenten el establecimiento de indicadores clave para la enseñanza de la disciplina (Scheldon et al., 2011).

$\mathrm{Al}$ realizar un recuento de las investigaciones seleccionadas sobre el marco conceptual de pertinencia en la educación superior, y considerando la tesis de la reproducción social y cultural de Bordieu y Passeron (2000) y el sistema de auto-legitimación, se puede inferir que, desde una postura epistémica crítica, se privilegiaron los métodos cualitativos de investigación y en un solo caso se acudió al enfoque mixto. En este rubro las categorías de análisis presentan concomitancia en elementos de la pertinencia como: equidad, responsabilidad social, flexibilidad, función social, competencias, calidad, función crítica y gestión educativa.

Desde una postura epistémica interpretativa, los hallazgos determinan que el enfoque metodológico que se privilegia es el cualitativo, seguido del método mixto de investigación; con respecto a las categorías abordadas en estas investigaciones, se destacan la evaluación institucional, las normas éticas y la vinculación laboral.

El menor número de investigaciones estuvo abordada a partir de una postura epistémica positivista; en este punto la pertinencia de la educación superior es examinada bajo enfoques cuantitativos de investigación, en los cuales se privilegian categorías que van desde la vinculación, las políticas educativas internas, las funciones sustantivas como la investigación a cargo de los académicos, la alineación de los programas educativos al servicio del sector productivo, la productividad y la formación de recursos humanos.

En el mismo tenor que el análisis anterior, los resultados de la producción científica en torno a la pertinencia en la formación profesional en turismo, arrojaron los siguientes hallazgos: el 50\% de las investigaciones se abordaron desde el positivismo científico privilegiando los enfoques cuantitativos de investigación, con categorías que van desde la vinculación de la industria con las IES que promueven programas de turismo, pertinencia del currículo dirigido a la empresa, la formación de recursos humanos con visión o perspectiva gerencial y el desarrollo de habilidades centradas en las necesidades de la industria.

El paradigma interpretativo fue el empleado por investigaciones que utilizaron enfoques de investigación cualitativo y mixto, privilegiando el primero. En este paradigma, las categorías elegidas fueron el diseño curricular ampliado, tendencias en la educación del turismo, la educación binaria (técnica-profesional) y el desarrollo social.

En ascenso, las investigaciones desde un paradigma crítico parten del enfoque mixto; las categorías de análisis que predominan son la responsabilidad social, la ética, los valores, la pertinencia, la percepción de los educadores y profesionales en turismo, las competencias, el pensamiento lógico, la interacción social, la orientación a la reflexión y acción liberadora de los contenidos curriculares y el desarrollo profesional.

Los autores europeos que han investigado el tema de la pertinencia en la formación profesional en turismo, lo han hecho principalmente desde tres paradigmas de investigación: el positivismo científico, la postura interpretativa y la teoría crítica. Sin embargo, el $68 \%$ de los trabajos fue motivado bajo la premisa de responder a las necesidades de la industria del turismo, lo cual significa que las IES se identifican con el enfoque economicista de la pertinencia de la educación superior. De manera complementaria, el 19\% de las investigaciones se enmarcan dentro de un enfoque social de la pertinencia; en lo que respecta al integral, el 13\% de las investigaciones se pueden enmarcar en este enfoque. El cuadro 4 da cuenta de los resultados sobre la producción científica en torno a la pertinencia en la formación profesional en turismo, los enfoques de investigación predominantes y el enfoque de pertinencia al que responden. 
Cuadro 4. Compendio de resultados

\begin{tabular}{|l|c|c|c|}
\hline \multicolumn{1}{|c|}{ Paradigmas de investigación } & $\begin{array}{c}\text { Enfoque de investigación } \\
\text { predominante }\end{array}$ & Enfoque de pertinencia & $\begin{array}{c}\text { Porcentaje de las } \\
\text { investigaciones }\end{array}$ \\
\hline Positivismo científico & Cuantitativo & Enfoque economicista & $68 \%$ \\
\hline Método interpretativo & Cualitativo y Mixto & Enfoque Social & $19 \%$ \\
\hline Teoría crítica & Mixto & Enfoque integral & $13 \%$ \\
\hline Total & & & $100 \%$ \\
\hline
\end{tabular}

Fuente: elaboración propia.

Lo anterior indica que, independientemente de la postura o paradigma de investigación que privilegia cada autor, para el caso europeo la pertinencia en la formación profesional turística encuentra su marco de acción alrededor del enfoque economicista.

Para el caso de Latinoamérica, los autores privilegian en sus investigaciones el enfoque social para analizar la pertinencia de la educación superior, mientras que, desde un enfoque economicista y un enfoque integral, se identificaron trabajos en iguales proporciones. El discurso de pertinencia social en las IES ya no es relevante (García, 2003; Fundora, 2010). Chomsky (2014) advierte, que el trabajo académico es un asalto neoliberal general a la población donde las universidades públicas avanzan por la vía de un modelo de negocio empresarial (Laval, 2004), donde se ha registrado en los últimos 30 años un aumento drástico en la proporción de personal administrativo en relación con el personal docente; hecho también verificado por Ginsberg (2011) el cual describe el estilo empresarial de administración y los niveles burocráticos multiplicados en las IES. Es necesario considerar que la investigación en turismo es muy joven en Latinoamérica, a pesar de los esfuerzos significativos en la producción para transformar la región en un núcleo de conocimiento turístico que contribuya a las instituciones y sus programas educativos (Picazo y Moreno, 2013a, 2013b).

\section{Consideraciones finales}

La Conferencia Mundial de la Educación Superior celebrada en 1998, constituye un referente al respecto de dos problemas en concreto: 1 . Incapacidad de la educación superior y de la formación para adaptarse a un mundo cambiante; 2. Compromiso insuficiente por parte de los gobiernos, especialmente en los países en desarrollo. Para 2008, con ocasión de los trabajos auspiciados por la UNESCO, acerca de la educación superior en América Latina y el Caribe, diez años después de la Conferencia Mundial, la situación no era diferente, y lo que es peor, nuevas limitaciones se habían detectado.

El tema de la pertinencia, otrora protagonista en la Conferencia Mundial, eclipsó en los debates posteriores, en donde el foco principal fue la evaluación de la calidad de los programas educativos de nivel superior y los modelos educativos de Norteamérica que dicho sea de paso se erigieron con relativo éxito en la formación de recursos humanos de élites que controlaban las grandes multinacionales. Siete años después, en 2015, el discurso ha cambiado y se publica acerca de la masificación de la educación superior bajo un modelo capitalista que responde a las demandas de la clase media-baja latinoamericana en aumento. El discurso de la pertinencia de la educación superior ya no es relevante, o al menos no es prioritario en los países de la misma región. 
La pertinencia de la educación superior debe comprender el trabajo y compromiso que las universidades llevan a cabo con la sociedad y el entorno en todo su conjunto, es decir sector social y empresarial.

Es un proceso de doble vía, desde la universidad y desde la sociedad; donde participan agentes externos y agentes internos que conjugan esfuerzos para consolidar una tarea central, su responsabilidad social.

En los procesos de evaluación institucional al interior de las IES, la calidad y la pertinencia no han recibido la misma atención; el predominio de la primera ha sido una constante para los organismos evaluadores.

Para ser pertinentes, las IES deben establecer varios compromisos, entre los que se destaca el establecimiento de múltiples tipos de vinculaciones entre agentes internos vs agentes externos. De esta manera, pertinencia es cumplir con la declaración del proyecto educativo institucional enunciado en objetivos, misión, visión y valores; generando resultados que sean relevantes para la sociedad, los cuales emanan del cumplimiento de sus metas.

Otro compromiso impostergable de las IES lo constituye el mejorar los procesos administrativos con el fin de equipararse a la velocidad y lo inédito de las situaciones emergentes que permitan comprender y resolver las nuevas exigencias. Un programa educativo pertinente en el siglo XXI, debe contar con marcos de acción transversal (que permita captar las múltiples percepciones de la vida humana) y horizontal (que permita la percepción de escenarios a largo plazo).

De tal manera, sigue abierto el debate de centrar el currículo en los requerimientos de la industria o centrarlo en el estudiante. La discusión realmente debería girar en torno al grado de flexibilidad para el diseño de modelos curriculares con capacidad de respuesta en varias direcciones; es decir, comunidad universitaria y sectores sociales, lo cual sería consistente en la discusión de pertinencia de la educación superior desde un enfoque social.
Comprender las IES como organizaciones modernas capaces de analizar la situación actual en la formación de profesionales para el turismo en el ámbito latinoamericano, es lo que podría constituir su razón de ser para valorar su pertinencia desde un enfoque integral. Diseñar escenarios a futuro que le permitan actuar en términos de docencia, planta física, planes de estudio, necesidades de investigación, vinculación laboral de egresados, calidad e impacto en las diversas comunidades hace parte de dicho enfoque.

Las IES pueden aprender de la industria turística su capacidad de transformación e innovación, pero recuperando y conservando el espíritu crítico y emancipador de la comunidad universitaria. No se trata de desprenderse de su carácter público, sino de incorporar las mejores prácticas empresariales respetando la postura socio-crítica en la formación de profesionales para el turismo. Las ciencias sociales tienen una larga tradición de pensamiento crítico, y desde la formación de estudiantes a nivel de pregrado hasta la investigación del más alto nivel, la academia debe incentivar la consolidación de puntos de vista propios, independientes y reflexivos.

Es urgente que los estudios realizados desde y para la actividad turística se lleven a cabo incorporando un aparato crítico desde las ciencias sociales, un verdadero fundamento teórico que permita acercarnos a la ciencia y generar conocimiento validado para el turismo. Se evidencia un vacío de conocimiento en lo que respecta al tema de la pertinencia en la formación de profesionales en turismo. Desde hace décadas se ha incursionado en temas afines desde otras disciplinas a partir de las cuales académicos e investigadores diseñaron planes de estudio alejados de la vocación turística del país.

Las reflexiones al respecto de la pertinencia en la formación de profesionales en turismo, deben comenzar con la iniciativa de los actores de la industria turística, en el sentido de admitir sobre los diversos componentes/contenidos que la integran; éstos se combinan para conformar una disociación que 
se traduce en propuestas curriculares variadas a cargo de las IES que forman para el turismo.

Es necesario abordar el estudio de la pertinencia a partir de un aparato teórico que permita analizar el discurso de los diversos actores que participan en la misión de formar profesionales en turismo. Independiente del nivel de estudios y el corte profesionalizante o de investigación de los programas educativos, se trata de dar una nueva cara a la investigación y por ende un impacto en los programas diseñados para la formación de profesionales en turismo.

Se ha identificado que la investigación sobre pertinencia en programas de formación en turismo a nivel superior en México ha sido poco documentada

\section{Referencias}

Airey, D. (2005), "Growth and development", en David Airey y John Tribe (eds.), An International Handbook of Tourism Education, Amsterdam, Elsevier, pp. 13-24.

Airey, D. y V. Middleton (1995), "Education and training in tourism in the UK", en Guidelines, núm. 3, Association for Tourism in Higher Education in the UK [The National Liaison Group for Higher Education in Tourism (NLG)].

Airey, D., J. Tribe, P. Benckendorff y H. Xiao (2014), "The managerial gaze: the long tail of tourism education and research", en Fournal of Travel Research, pp. 1-13.

Alcántara, A. y J. Zorrilla (2010), "Globalización y educación media superior en México. En busca de la pertinencia curricular", en Perfiles Educativos, Vol. XXXII, núm. 127, pp. 38-57.

Almonacid, P., Montes, I. \& Vásquez, J. (2009), “Un análisis factorial para evaluar la pertinencia de un programa académico desde la perspectiva de los graduados: un estudio de caso", en Ecos de Economía, núm. 29, octubre, pp. 1-29. en artículos científicos; pese a que el diseño o el rediseño curricular de un plan de estudios, exige elaborar estudios de pertinencia que los avale (Plan Nacional de Desarrollo 2013-2018).

Para finalizar, se vislumbran dos líneas de investigación: en primer lugar la discusión sobre la importancia atribuida a la pertinencia de la educación superior con un interés meramente administrativo por encima del quehacer científico; y en segundo, dado el reducido número de estudios sobre pertinencia en la formación de profesionales en turismo en México, el tema abordado desde un enfoque social encuentra un campo fértil para su desarrollo conceptual y práctico.
Arellano, P. y A. Castro (2013), "Análisis de la situación de la formación profesional desde el punto de vista de sus protagonistas", en Educatio Siglo XXI, Vol. 31, núm. 2, pp. 255-276.

Avcikurt, C. (2000), "Education and training in tourism in Turkey: problems and prospects", en Research Gate, pp. 282-297.

Avcikurt, C., B. Alper y S. Geyik (2009), "Education and training of tourist guides in Turkey", en Management and Education, vol. V, núm. 1.

Ayikoru, M., J. Tribe y D. Airey (2009), "Reading tourism education. Neoliberalism unveiled", en Annals of Tourism Research, vol. 36, núm. 2, pp. 191-221.

Banco Interamericano de Desarrollo (BID) (2009), Estrategia para el fortalecimiento del capital humano del sector, con base en las competencias de las personas, México, BID [Sector Turismo, CONOCER].

Beltrán,J., E. Iñigo y A. Mata (2014), “La responsabilidad social universitaria, el reto de su construcción permanente", en Revista Iberoamericana de Educación 
Superior (RIES), México, UNAM-IISUE/Universia, vol. V, núm. 14, pp. 3-18.

Bordieu, P. \& Passeron, J. (2000), Reproduction in education, society and culture [2nd. ed., trad. de Richard Nice. London], Londres, SAGE Publications.

Botterill, D. (1996), "Making connection between industry and higher education in tourism", en Guidelines, núm. 5, Association for Tourism in Higher Education in the UK [The National Liaison Group for Higher Education in Tourism (NLG)].

Botterill, D. y J. Tribe (2000), "Benchmarking and the higher education curriculum", en Guidelines, núm. 9, Association for Tourism in Higher Education in the UK [The National Liaison Group for Higher Education in Tourism (NLG)].

Buendía, A. (2014), La FIMPES y la mejora de la calidad de instituciones privadas. Cambio, prestigio y legitimidad. Tres estudios de caso (1994-2004), México, Asociación Nacional de Universidades e Instituciones de Educación Superior (ANUIES).

Busby, G. (2003), "The concept of sustainable tourism within the higher education curriculum: a British case study", en Fournal of Hospitality, Leisure, Sport \& Tourism Education, vol. 2, núm. 2, pp. 48-58.

Busby, G. y D. Fiedel (2001), "A contemporary review of tourism degrees in the United Kingdom”, en Fournal of Vocational Education and Training, 53 (4), pp. 501-522.

Busby, G. y R. Huang (2012), "Integration, intermediation and tourism higher education: conceptual understanding in the curriculum", en Tourism Management, vol. 33, pp. 108-115.

Camarena, B. y D. Velarde (2010), "Educación superior y mercado laboral: vinculación y pertinencia social ¿Por qué? y ¿para qué?”, en Estudios Sociales, Revista de Investigación Científica, núm. Especial, enero.

Collado, L., L. Medina, A. Herrera y M. Moreno (2013), "Innovación curricular en turismo: un campo de estudio y referentes para la formación universitaria", en Revista Actualidades Investigativas en Educación, vol. 13, núm. 1, pp. 1-39.

Cooper, C. (2012), “Contemporary tourism education: notes from the frontline", en Tej Singh (ed.), Critical Debates in Tourism, cap. 8, Toronto, Channel View Publications.

Corzo, L. y N. Marcano (2009), "Pertinencia del currículo de las instituciones de educación superior: un estudio cualitativo desde la teoría fundamentada”, en Multiciencias, Venezuela, Universidad del Zulia, vol. 9, núm. 2, mayo-agosto, pp. 149-156.

Chavoya, M. y S. Reynaga (2011), "Las licenciaturas en Jalisco, México: crecimiento, diversificación y complejización de la educación superior y su impacto en la pertinencia", en Revista Espacio Abierto, vol. 20, núm. 2, pp. 289-311.

Chomsky, N. (2014), "El trabajo académico, el asalto neoliberal a las universidades y cómo debería ser la educación", recuperado en: Ssociólogos, <http:// ssociologos.com/2014/03/13/noam-chomskyel-trabajo-academico-el-asalto-neoliberal-a-lasuniversidades-y-como-deberia-ser-la-educacion/?utm_ content=buffer8ce99\&utm_medium=social\&utm source=twitter.com\&utm_campaign=buffer $>$ [Consulta: diciembre 2015].

Coronado, M. y E. Estévez (2016), "Pertinencia social de una universidad pública en México, desde la visión de estudiantes, empleadores y docentes", en Revista Iberoamericana de Educación Superior (RIES), México, UNAMIISUE/Universia, vol. VII, núm. 20, pp. 172-189.

Craig-Smith, S. y P. Ding (2007), "Tourism degree education in Australia and its relevance to China", en Fournal of Teaching in Travel \& Tourism, vol. 7, núm. 1, pp. 45-62.

De Ketele, J. (2008), "La pertinencia social de la educación superior”, en La educación superior en el mundo. La Globalización y los mercados: retos de la educación superior, Nuevos/ Global University Network for Innovation (GUNI), pp. 55-61.

Días-Sobrinho, J., A. Stubrin, E. Martín, L. González, O. Espinoza y P. Goergen (2008), "Calidad, pertinencia y responsabilidad social de la universidad latinoamericana y caribeña", en Tendencias de la educación superior en América Latina, 61, pp. 87-112. 
Eber, S. (2003), "Integrating sustainability into the undergraduated curriculum: leisure and tourism", en Guidelines, núm. 10, UK, Association for Tourism in Higher Education in the UK/The National Liaison Group for Higher Education in Tourism (NLG).

Ernawati, D. y P. Pearce (2003), "Tourism courses at the higher education level in Indonesia: the perspectives of the stakeholders", en Fournal of Teaching in Travel \& Tourism, vol. 3, núm. 2, pp. 1-18.

Escobar, M. (2003), "La sociedad del riesgo global" [Crítica de Libro], en Revista Española de Investigaciones Sociológicas, núm. 101/3, pp. 279-303.

Fidgeon, P. (2010), "Tourism education and curriculum design: ¿A time for consolidation and review?", en Tourism Management, vol. 31, pp. 699-723.

Fundora, G. (2010), "La educación superior avanzada: calidad-equidad-pertinencia: tasas de coberturas terciarias en América Latina y El Caribe", en Pedagogía Universitaria, vol. 15, núm. 1, pp. 41-57.

García Guadilla, C. (2003), "Balance de la década de los '90 y reflexiones sobre las nuevas fuerzas de cambio en la educación superior", en Marcela Mollis (comp.), Las universidades en América Latina: ¿Reformadas o alteradas? La cosmética del poder financiero, Buenos Aires, CLACSO.

García Guadilla, C. (1997), "El valor de la pertinencia en las dinámicas de transformación de la educación superior en América Latina”, en La educación superior en el siglo XXI. Visión de América Latina y el Caribe, Caracas, Instituto Internacional para la Educación Superior en América Latina y el Caribe/Organización de las Naciones Unidas para la Educación, la Ciencia y la Cultura, 2.

Garrocho, C. y G. Segura (2012), "La pertinencia social y la investigación científica en la universidad pública Mexicana", en CIENCIA ergo sum, vol. 19-1, marzojunio, Toluca, Universidad Autónoma del Estado de México, pp. 24-34.

Gibbons, M. (1998), Pertinencia de la educación superior en el siglo XXI, Washington, The World Bank.

Ginsberg, B. (2011), The fall of the faculty: the rise of the alladministrative university and why it matters, Oxford, Oxford
University Press.

Gómez, S. (2012), Educación superior e investigación turística. Retos, problemas y desilusiones, Guadalajara, Universidad de Guadalajara, Centro Universitario de Ciencias Económico Administrativas/Editorial Página Seis.

Gómez, V. (2009), "Desempeño laboral del Licenciado en turismo de la Universidad de Oriente, según la opinión del sector empresarial turístico privado de la Isla de Margarita, Estado Nueva Esparta”, en Gestión Turística, núm. 11, pp. 41-68.

Gómez, V. (1998), "Hacia una agenda sobre la pertinencia de la educación superior en Colombia”, en Políticas y estrategias para la transformación de la educación superior en América Latina y el Caribe. Hacia una agenda de la educación superior en Colombia, Santafé de Bogotá, ASCUN/UNESCO/CRESALC.

Goodenough, R. y S. Page (1993), "Planning for tourism and training in the 1990s: Bridging the gap between industry and education", en Fournal of Geography in Higher Education, vol. 17, núm. 1, pp. 57-72.

Gottifredi, J. C. (1996). "La pertinencia de la educación superior", en La transformación universitaria en vísperas del tercer milenio, Montevideo, Memorias del Simposio AUGM/SM/UDUAL GRESALG/UNESCO, pp. 13-16.

Gunn, G. (1998), "Issues in tourism curricula”, en fournal of Travel Research, vol. 36, núm. 4, pp. 74-77.

Holloway, C. (1995), "Towards a core curriculum for tourism: a discussion paper", en Association for Tourism in Higher Education (ATHE) E The National Liaison Group for Higher Education in Tourism (NLG).

Horng, J. y M. Lee (2005), "Tourism and hospitality higher education in Taiwan: past, present, and future", en fournal of Teaching in Travel \& Tourism, 5(3), pp. 167-196.

Hunziker, W. y K. Krapf (1942), Fundamentos de la teoría general del turismo, Zurich, Poligraphic publishing house.

Jafari, J. (2005), "El turismo como disciplina científica", en Política y Sociedad, vol. 42, núm. 1, pp. 39-56.

Kemmis, S. (1993), El curriculum, más allá de la teoría de la reproducción [2da. Edición], Madrid, Ediciones Morata [Colección Pedagogía].

Koh, K. (1995), "Designing the four-year tourism 
management curriculum: a marketing approach", en Fournal of Travel Research, 33, pp. 68-72.

Laval, C. (2004), La escuela no es una empresa: el ataque neoliberal a la enseñanza pública, Barcelona, Editorial Paidós Ibérica.

Lombana, J. (2012), "Pertinencia de la educación en la competitividad", en ZONA PRÓXIMA, Revista del Instituto de Estudios en Educación Universidad del Norte, núm. 16, pp. 68-85.

López, A. (2007), "Pertinencia y alcance del análisis organizacional para el estudio del curriculo", en Administración y Organizaciones, 18, año 9, pp. 63-79.

Malagón, L. (2009a), "La pertinencia curricular: un estudio en tres programas universitarios", en Educación y Educadores, vol. 12, núm. 1, pp. 11-27.

Malagón, L. (2009b), "La pertinencia en la educación superior: una mirada crítica", Ponencia publicada en memoria electrónica, recuperado en: <http://www. congresoretosyexpectativas.udg.mx/Congreso\%203/ Mesa\%203/Mesa3_21.pdf> [Consulta: diciembre 2014].

Malagón, L. (2006), "Perspectiva economicista en la vinculación universidad-sociedad", en Uni-pluril versidad, vol. 6, núm. 2, pp. 37-45.

Malagón, L. (2003), "La pertinencia en la educación superior. Elementos para su comprension", en Revista de la Educación Superior, vol. 32 (3), núm. 127, pp. 113-134.

Martínez, R. (2003), "La inserción laboral de los universitarios a través de las prácticas en las empresas", en Revista Española de Investigaciones Sociológicas, núm. 101, pp. 228-254.

Marúm, E. y V. Rosario (2001), "Tendencias de las profesiones del área económico-administrativa”, en Perfiles Educativos, vol. 23, núm. 93, pp. 44-58.

Mayaca, M. y J. Akama (2007), "Systems approach to tourism training and education: the Kenyan case study", en Tourism Management, vol. 28, pp. 298-306.

Méndez, E. (2005), "La pertinencia como requisito para la calidad en educación superior. La planeación institucional y el compromiso como condición esencial para el desarrollo del posgrado", en Revista Iberoamericana de Educación, núm. 36/3.
Mora, M. y O. de Oliveira (2012), "Las vicisitudes de la inclusión laboral en los albores del siglo XXI: trayectorias ocupacionales y desigualdades sociales entre jóvenes profesionistas mexicanos", en Estudios Sociológicos, vol. 30, núm. 88, pp. 3-43.

Ministerio de Educación de Argentina (2013), Lineamientos de mejora para la formación de recursos humanos en turismo, Buenos Aires, Ministerio de Turismo, Secretaría de Políticas Universitarias.

Mungaray, A., M. Ocegueda, P. Moctezuma y J. Ocegueda (2016), "La calidad de las universidades públicas estatales de México después de 13 años de subsidios extraordinarios", en Revista de la Educación Superior, vol. XLV (1), núm. 177, enero-marzo, pp. 67-93.

Navarro, A., M. Álvarez y J. Gottifredi (1997), "Pertinencia social de la universidad. Una propuesta para la construcción de la imagen institucional", en Educación Superior y Sociedad, vol. 8, núm. 2, pp. 75-96.

Naidorf, J., P. Giordana y M. Horn (2007), "La pertinencia social de la universidad como categoría equívoca”, en Nómadas, núm. 27, pp. 22-33.

Nieto, M. (2014), "Excelencia o pertinencia: para dónde van las ciencias sociales en la Universidad de los Andes", en Revista de Estudios Sociales, núm. 50, pp. 174-177.

Núñez, J. (2010), "Pertinencia de la educación rural venezolana y latinoamericana", en Revista Iberoamericana de Educación, núm. 52, pp. 1-14.

Ordorika, I. (2015), "La educación superior en el XIII Congreso Nacional de Investigación Educativa", Editorial, en Revista de la Educación Superior, vol. XLIV (4), núm. 176, pp. 7-11.

Organización Mundial del Turismo (OMT) (2009), Estudio de la oferta y la demanda de formación de los recursos humanos en el sector turístico en América Latina, México, Informe Final, Secretaría General Iberoamericana.

Organización para la Cooperación y el Desarrollo Económico (OCDE) (1997), Reviewes of national policies for education: Mexico, París, OECD Publishing.

Orozco, L. (2010), "Calidad académica y relevancia social de la educación superior en América Latina", en Revista Iberoamericana de Educación Superior (RIES), México, 
IISUE-UNAM/Universia, vol. 1, núm.1, pp. 24-36.

Osorio, E., S. Martínez y E. Contreras (2010), "Estudio de pertinencia social del programa educativo de Químico de la Universidad Autónoma del Estado de México", en Revista Educación Química, 21 (1), pp. 22-27. Plan Nacional de Desarrollo (2013-2018), Gobierno de la República. Estados Unidos Mexicanos, recuperado en <http://www.sev.gob.mx/educacion-tecnologica/ files/2013/05/PND_2013_2018.pdf> [Consulta: mayo 2016].

Pérez, J. y M. Merino (2010), Definición de pertinencia, recuperado en <http://definicion.de/pertinencia/> [Consulta: octubre 2016].

Pérez, L. (2007), "Pertinencia y extensión universitaria en el contexto de la nueva universidad cubana", en Revista Pedagogía Universitaria, vol. XII, núm. 1, pp. 1-10.

Perrenoud, P. (2005), "Diez nuevas competencias para enseñar", en Educatio Siglo XXI, vol. 23, pp. 223-229.

Pernas, M., S. Sierra, J. Fernández, E. Miralles y J. Diego (2009), "Principios estratégicos de la educación en Ciencias de la salud en Cuba (II): la pertinencia", en Educación Médica Superior, 23 (2).

Picazo, P. y S. Moreno (2013a), "Difusión de la investigación científica iberoamericana en turismo", en Estudios y Perspectivas en Turismo, vol. 22, pp. 828-853.

Picazo, P. y S. Moreno (2013b), "Difusión de la investigación científica en turismo. El caso de México", en El Periplo Sustentable, núm. 24, enero-junio, pp. 7-40.

Picazo, P. y S. Moreno (2012), "Difusión de la investigación científica de turismo en Chile y Argentina", en Gestión Turística, núm. 18, pp. 9-45.

Ring, A., A. Dickinger y K. Wöber (2009), "Designing the ideal undergraduated program in tourism: expectations from industry and educators", en fournal of Travel Research, vol. XX, núm. X, pp. 1-16.

Salgado, M. (2004), "Pertinencia y responsabilidad social de las universidades en el proceso de construcción de la paz”, en Revista Iberoamericana de Educación, pp. 1-10.

Schleicher, A. (2008), "A new strategy for assesing adult competencies", en International Review of Education, vol. 54, núm. 5/6 [Special Issue: Literacy education for all: challenges and prospects], pp. 627-650.

Sheldon, P., Fesenmaier, D. \& Tribe, J. (2011), "The Tourism Education Futures Initiative (TEFI): Activating Change in Tourism Education", en fournal of Teaching In Travel \& Tourism, 11(1), pp. 2-23.

Solnet, D., R. Robinson y C. Cooper (2007), "An industry partnerships approach to tourism education", en Journal of Hospitality, Leisure, Sport \& Tourism Education, vol. 6 , núm. 1.

Tan, J. y D. Morgan (2001), "Relevance and quality in Australian tourism higher: educator and professional views", en Fournal of Teaching in Travel \& Tourism, 1(1), pp. 59-78.

Tribe, J. (2005), "Tourism, knowledge and the curriculum", en David Airey y Jhon Tribe (eds.), An International Handbook of Tourism Education, Oxford, Elsevier, pp. 47-60.

Tribe, J. (2005a), “Overview of research”, en David Airey y John Tribe (eds.), An International Handbook of Tourism Education, Oxford, Elsevier, pp. 25-43.

Tribe, J. (2002), "The philosophic practitioner", en Annals of Tourism Research, vol, 29 (2), pp. 338-357.

Tribe, J. (2001), "Research paradigms and the tourism curriculum", en Fournal of Travel Research, SAGE Publications, vol. 39, pp. 442-448.

Tünnermann, C. (ed.) (2008), La educación superior en América Latina y el Caribe: diez años después de la Conferencia Mundial de 1998, Bogotá, Pontificia Universidad Javeriana/UNESCO, IESALG.

Tünnermann, C. (2007), La universidad necesaria para el siglo XXI, Managua, HISPAMER.

Tünnermann, C. (2006), "Pertinencia y Calidad de la Educación Superior”. Lección Inaugural, Guatemala. Recuperado en: <http://biblio2.url.edu.gt:8991/ libros/leccion\%20inaugural2006texto.pdf> [Consulta: enero 2016].

Tünnermann, G. y M. de Souza (2003), "Desafíos de la universidad en la sociedad del conocimiento, cinco años después de la Conferencia Mundial sobre Educación Superior", UNESCO Forum Occasional Paper Series, núm. 4/S. 
Tünnermann C. (2000), "Pertinencia social y principios básicos para orientar el diseño de políticas de educación superior", en Educación Superior y Sociedad, vol. 11, núm. 1 y 2, pp. 181-196.

Universidad La Salle / Secretaría de Turismo / Cestur /CONACyT (2012), Alineación de los programas de estudio de educación turística a las necesidades del campo laboral, México, Universidad La Salle/ Secretaría de Turismo/ Centro de Estudios Superiores en Turismo/ Consejo Nacional de Ciencia y Tecnología.

United Nations Educational, Scientific and Cultural Organization (UNESCO) (1998), La educación superior en el siglo XXI. Visión y acción. La contribución de la educación superior al sistema educativo en su conjunto, Documento final sobre la Conferencia Mundial de la Educación Superior, París, UNESCO.
Urry, J. y J. Larsen (2011), The Tourist Gaze 3.0, SAGE Publications.

Vessuri, H. (1996), "Pertinencia social de la educación superior latinoamericana a finales del siglo XXI", en Nueva Sociedad, núm. 146, nov-dic, pp. 102-107.

Vessuri, H. (2008), "De la pertinencia social a la sociedad del conocimiento", en La educación superior en América Latina y el Caribe: diez años después de la Conferencia Mundial de 1998, Bogotá, Pontificia Universidad Javeriana/ UNESCO, IESALC.

Walmsley, A. (2012), "Pathways into tourism higher education", en Fournal of Hospitality, Leisure, Sport \& Tourism Education, vol. 11, núm. 2, pp. 131-139.

Zagonari, F. (2009), "Balancing tourism education and training", en International Fournal of Hospitality Management, vol. 28, pp. 2-9. 